\title{
FRESH EYES: empowering junior doctors as agents for change
}

\author{
Author: Clare Philliskirk
}

\section{Aims}

> It is recognised that we are at our most observational, reflective and questioning in the first few days of being in a new environment. This is a time when we are most likely to identify opportunities to make improvements.

> Junior doctors (JDs), with their regular job rotations and unique experiences of the healthcare system, are ideally placed as agents for change but are in the large part under-valued and under-utilised.

> By encouraging and empowering junior doctors to record these initial observations and supporting them with projects, we can have a positive impact on their engagement and morale, while driving quality improvement forward.

\section{Methods}

A baseline survey was conducted to assess how well JDs working in the acute medical unit (AMU) in Birmingham City Hospital feel they are involved and supported in quality improvement, and how well they feel they are listened to. Data was also collected regarding the number and characteristics of completed quality improvement projects over the past 12 months.

An online data collection application (FRESH EYES) was designed and produced for JDs to complete during the first week of their rotation in the AMU in April 2018.

The JDs will be invited to take part in the project on a voluntary basis. Those who agree to participate will meet 2 weeks into their rotation in a focus group and will be supported to work together to develop quality improvement projects. They will further be supported with meetings every month for the remainder of their rotation.

A follow-up survey will be completed at the end of the rotation, as well as data about the quality improvement projects undertaken.

\section{Results}

Results are pending and will not fully be available until August 2018.

Author: Royal College of Physicians chief registrar, Sandwell and West Birmingham NHS Trust, UK

\section{Conclusion}

Various industries have used the 'fresh eyes' concept to identify opportunities to make improvement for a number of years. The Francis report in 2013 stated that JDs are the 'eyes and ears' of the NHS. By recognising that JDs can play a vital role in questioning how things are done, and supporting and empowering them to make improvements, we can not only enhance their engagement and morale but also enable meaningful quality improvement to take place.

\section{Conflict of interest statement}

None. 